\title{
A Low Power mm-wave Oscillator Using Power Matching Techniques
}

\author{
Lianming Li, Patrick Reynaert, Michiel Steyaert \\ KU Leuven ESAT-MICAS, Leuven, Belgium \\ \{Lianming.Li, Patrick.Reynaert, Michiel.Steyaert\}@esat.kuleuven.be
}

\begin{abstract}
A low power low voltage 90nm CMOS mmwave oscillator using a power matching technique is presented. The oscillator uses an inductive divider to create impedance matching for the amplifier. With this technique, the effect of the varactor loss on the phase noise is reduced and the oscillator power efficiency is increased. The proposed oscillator achieves a phase noise of $-95 \mathrm{dBc} / \mathrm{Hz}$ at $1 \mathrm{MHz}$ offset from $64 \mathrm{GHz}$ carrier, consuming $3.16 \mathrm{~mW}$ from a $0.6 \mathrm{~V}$ supply voltage. The figures-of-merit are FOM -186 and $\mathrm{FOM}_{\mathrm{T}}-185$ respectively. The tuning range is from $61.1 \mathrm{GHz}$ to $66.7 \mathrm{GHz}$ and the measured output power is about -14 dBm.
\end{abstract}

Index Terms - LC oscillator, millimeter-wave oscillators, power matching, quality factor, VCO.

\section{INTRODUCTION}

With the increasing requirements for higher data-rate, the $60 \mathrm{GHz}$ band is a very good candidate for the applications like network connection, HDTV etc [1]. Considering the fabrication cost and the scaling benefits, CMOS technology can be used to design mm-wave circuits [2]. As the key component of the frequency synthesizer, the oscillator design has a lot of implications for the system architecture and frequency planning [3]. As mentioned in [4], there are many challenges for mm-wave oscillators design ranging from the large tuning range, very high frequency to low supply voltage etc.

Up till now, several fundamental mm-wave oscillators have been designed using different bulk CMOS or SOI processes [5]-[9]. However achieving a large tuning range and low phase noise with good power efficiency still remains a challenge. In this paper we proposed to use power matching techniques to achieve better power efficiencies.

Basically, $L C$ oscillators can be understood as tuned amplifiers connected in a positive feedback topology. For mm-wave oscillators, the low gain of amplifiers at high frequencies limits the oscillator performance. To overcome the limitations, the proposed oscillator employs an inductor divider to achieve power matching between the transistor and the $L C$ tank. Experimental results show that a power efficient oscillator with a large tuning range and good phase noise can be achieved using this technique.

\section{DESIGN OF POWER MATCHING CiRCUIT}

A realistic oscillator typically consists of two parts: 1) an $L C$ tank for frequency selectivity and frequency tuning and 2) an amplifier connected in positive feedback to compensate the inevitable loss of a physical $L C$ tank. For mm-wave oscillators, the varactor and the amplifier are important noise sources.

For the $L C$ tank, the important figure-of-merit is the tank quality factor $(Q)$. Because the oscillator phase noise performance relies considerably on the tank $Q$, the oscillator designer should first maximize the tank $Q$ as high as possible. For $\mathrm{GHz}$ oscillators, the tank $Q$ is typically limited by the inductor loss. However in the mmwave range, because the quality factor is frequency dependent, the tank $Q$ is limited by the varactor loss. Moreover, for the large tuning range, the varactor capacitance to the parasitic capacitance ratio should be sufficiently large, making it very difficult to achieve a good phase noise in mm-wave application.

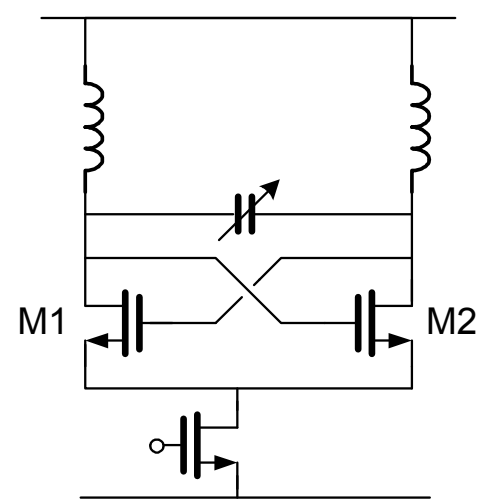

Fig. 1 The common differential oscillator structure 
As the loss compensating element, the amplifier is the other important part of the oscillator. It will set limits to the frequency tuning range and the phase noise performance improvement. Because the operating frequency is close to the transistor intrinsic frequency $f_{\mathrm{T}}$ and $f_{\text {MAX }}$, the amplifier power efficiency is typically poor.

In the common differential oscillator structure, shown in Fig.1, the gate of one transistor and the drain of the other are usually connected together. From an amplifier point of view, the drain impedance of transistor $M 1$ is the driving source impedance of transistor M2. To achieve an amplifier with optimum performance, one has to create a conjugate match between the drain impedance and the gate impedance. As shown in Fig. 2, in this particular 90nm CMOS technology, the intrinsic drain and gate real parallel impedance of a transistor biased in optimum conditions are respectively 473 and $2.34 \mathrm{k}$ at $60 \mathrm{GHz}$. Considering the loading effect from the $L C$ tank, the impedance at the transistor drain point is reduced further, leading to a severe impedance mismatch. Therefore, the working condition of the amplifier part in the oscillator is far from the optimum region, and the power efficiency of the amplifier suffers as a result.

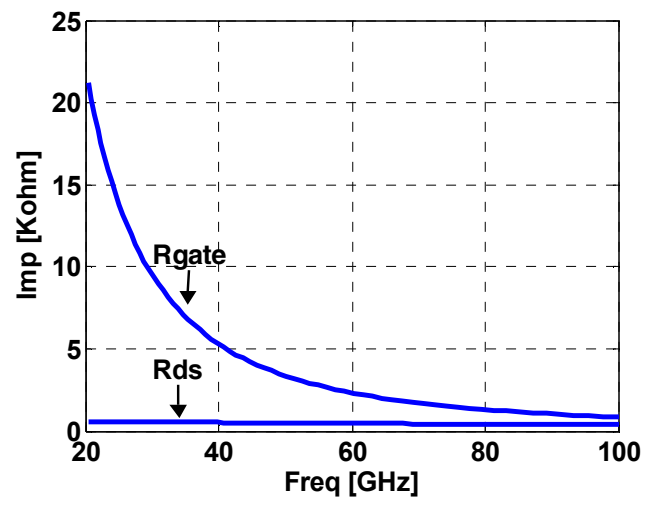

Fig. 2 Transistor gate and drain resistance $(2.34 \mathrm{k}$ and $473 \mathrm{ohm}$ respectively at $60 \mathrm{GHz})$

With the above problems in mind, an oscillator with an inductive divider power matching amplifier is proposed. Different from [4], the inductive divider of this work focuses on the matching of the transistors and reduces the influence of the varactor loss on the phase noise, thus improving power consumption and phase noise performance.

The proposed oscillator with the cascode buffer amplifier is shown in Fig.3. The tail current source is eliminated for voltage headroom and noise issues [4]. The differential oscillator tank comprises NMOS in nWell varactor and 2 inductors: $L 1$ and $L 2$. $L 1$ and $L 2$ function as the inductor divider, which is an impedance boosting vehicle. With the help of the inductor divider, the impedance is boosted up from the drain side to the gate side. In this way, the impedance match is realized, at least in the signal crossing point, which is the sensitive point for phase noise performance [11]. As a result, a higher power efficient amplifier is realized. Accordingly the signal amplitude at the gate side is larger than the drain side. In other words, the slope of the gate voltage is increased because the period of the drain and gate voltage signal is the same. The increased slope of the gate voltage in turn improves the slope of injection current at the drain point. Based on (1) [12], the increased slope is beneficial to the phase noise performance:

$$
\theta_{n}^{2}=\frac{2 \pi}{T} \cdot v_{n} \cdot \frac{d t}{d V_{A}}
$$

With $d V_{\mathrm{A}} / d t$ the signal slope at the crossing point, $T$ the signal period, ${ }_{n}$ the noise induced phase variation. (1) can be understood as follows: with higher signal slopes, a noise source with a certain value $v_{\mathrm{n}}$ has less time to convert into phase noise, thus improving the phase noise performance.

Considering the above benefits, the varactor is connected to the transistor drain side to reduce its effect on the phase noise. Such arrangement also has the advantage of achieving higher operating frequency because of the distributed effect.

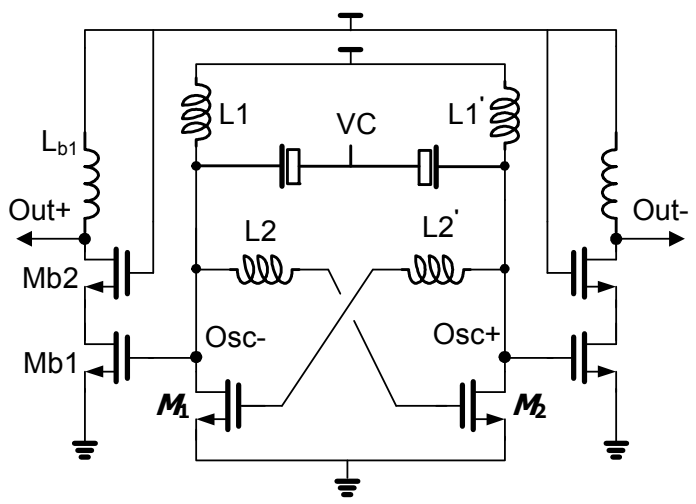

Fig. 3 The schematic of the proposed oscillator

Calculations indicate that the effect of the transistor parasitic capacitance on the oscillation frequency is enlarged because of the inductor divider. But with the improved amplifier efficiency, the transistor size of this proposed oscillator is reduced more than half compared with the previous oscillator [4], and so is the power consumption. So this structure will not have any negative effect on the tuning range, which is confirmed by the measurement results. 
TABLE I

PERFORMANCE SUMMARY OF STATE-OF-ART MM-WAVE OSCILLATORS

\begin{tabular}{|c|c|c|c|c|c|c|c|c|}
\hline VCO[Ref] & $\begin{array}{c}\text { FO } \\
\text { (GHz) }\end{array}$ & $\begin{array}{l}\text { FTR } \\
(\%)\end{array}$ & $\begin{array}{l}\text { PNoise } \\
\text { (dBc/Hz) }\end{array}$ & $\begin{array}{l}\text { PDISS } \\
(\mathrm{mW})\end{array}$ & $\begin{array}{l}\text { Vdd } \\
\text { (V) }\end{array}$ & $\begin{array}{c}\text { FOM } \\
\text { (dBc/Hz) }\end{array}$ & $\begin{array}{c}\text { FOM }_{\mathrm{T}} \\
(\mathrm{dBc} / \mathrm{Hz})\end{array}$ & Tech. \\
\hline H. Wang [5] & 49.5 & 2.21 & $-99.7 @ 1 M$ & 13 & 1.3 & -182.4 & -169.3 & 0.25 um CMOS \\
\hline M. Tiebout [6] & 51.2 & 1.39 & $-85 @ 1 \mathrm{M}$ & 1.0 & 1 & -179.2 & -162 & $0.12 \mathrm{um} \mathrm{CMOS}$ \\
\hline F. Ellinger [7] & 56.5 & 14.7 & $-92 @ 1 M-$ & 21.0 & 1.5 & -173.8 & -177.3 & 90nm SOI \\
\hline C. Cao [8] & 56.5 & 10.27 & 108@10M & 9.8 & 1.5 & -173.1 & -173.4 & $0.13 u m$ CMOS \\
\hline D. D. Kim [9] & 70.2 & 9.55 & $-106.1 @ 10 \mathrm{M}$ & 5.4 & 1.2 & -175.8 & -175.4 & $65 \mathrm{~nm} \mathrm{SOI}$ \\
\hline \multirow{2}{*}{ Borremans [10 } & 62.1 & 10 & $-95 @ 1 \mathrm{M}$ & 3.9 & 1 & -185 & -185 & \multirow{2}{*}{0.13 um CMOS } \\
\hline & 59.1 & 10.2 & $-91 @ 1 \mathrm{M}$ & 3.9 & 1 & -180.5 & -180.7 & \\
\hline \multirow{2}{*}{ L. Li [4] } & 58.4 & 9.32 & $-90 @ 1 \mathrm{M}$ & 8.1 & 0.7 & -176 & -176 & \multirow{2}{*}{ 90nm CMOS } \\
\hline & 61.7 & 4.81 & $-90 @ 1 M$ & 1.2 & 0.43 & -185 & -178.6 & \\
\hline This work & 64 & 8.75 & $-95 @ 1 M$ & 3.16 & 0.6 & -186 & -185 & 90nm CMOS \\
\hline
\end{tabular}

a. FOM=PNoise- $20 \log \left(f_{0} / \Delta f\right)+10 \log \left(P_{D I S S} / 1 \mathrm{~mW}\right)$

b. $\mathrm{FOM}_{\mathrm{T}}=$ PNoise $-20 \log \left(\left(f_{0} / \Delta f\right) *(F T R / 10)\right)+10 \log \left(P_{\text {DISS }} / 1 \mathrm{~mW}\right)$

\section{CHIP FABRICATION AND MEASUREMENT RESULtS}

The oscillator is realized in a 90nm CMOS process, and the chip micrograph is shown in Fig. 4. The inductors $L 1$ and $L 2$ in Fig. 3 are realized using a differential 3 turns inductor. The winding of this inductor is designed to make use of the positive magnetic coupling. Because of 3 turns coupling effect, a compact $L C$ tank could be realized. This area is about $110 \times 80 \mathrm{um}^{2}$. For the design of the inductor, special considerations are devoted to the balance between the self resonance frequency and the losses of internal inductors $L 2$. The reason is that the parasitic capacitance will limit the tuning range while the loss will reduce the signal slope.

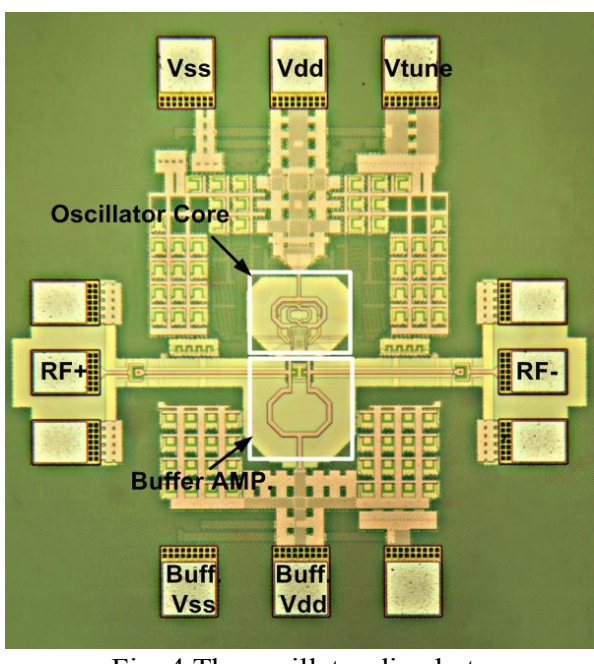

Fig. 4 The oscillator die photo

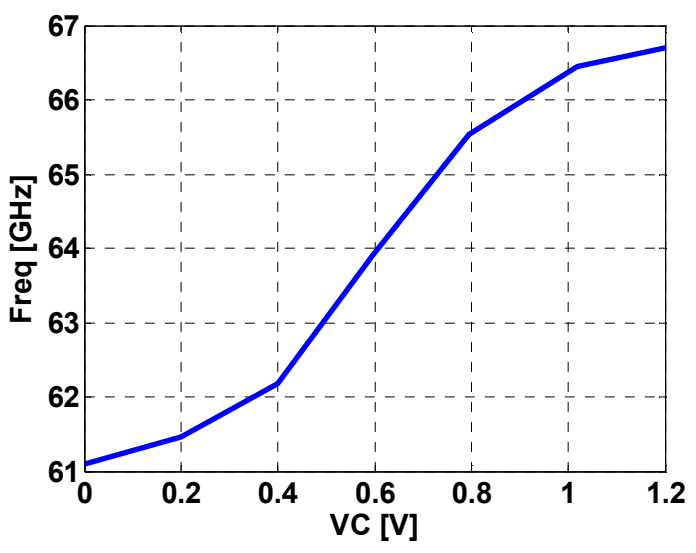

Fig. 5 The tuning curve of the oscillator

The oscillator works at a $0.6 \mathrm{~V}$ supply voltage, consuming a power consumption of only $3.16 \mathrm{~mW}$. The tuning curve of the oscillator is given in Fig. 5. With the tuning voltage ranging from 0 to $1.2 \mathrm{~V}$, the oscillator achieves a tuning range about $5.6 \mathrm{GHz}$ (from $61.1 \mathrm{GHz}$ to $66.7 \mathrm{GHz}$ ). The phase noise is measured using the delay line method, which is an accurate and reliable phase noise measurement method for free running oscillators. The phase noise curve at $64 \mathrm{GHz}$ is shown in Fig. 6. At $1 \mathrm{MHz}$ offset, the phase noise is about $-95 \mathrm{dBc} / \mathrm{Hz}$. Because of the pole effect of the delay line, the results above $5 \mathrm{MHz}$ offset are not accurate. The phase noise curve across the tuning range is shown in Fig. 7. Across the tuning range, the output power is about $-14 \mathrm{dBm}$. Table I offers the performance summary of the state-of-art mm-wave oscillators. To make the performance comparison, two 
figure-of-merits are used. At $64 \mathrm{GHz}$ carrier, the oscillator achieves a FOM and $\mathrm{FOM}_{\mathrm{T}}$ of -186 and -185 respectively. To the best knowledge of the authors, this advances the state-of-art.

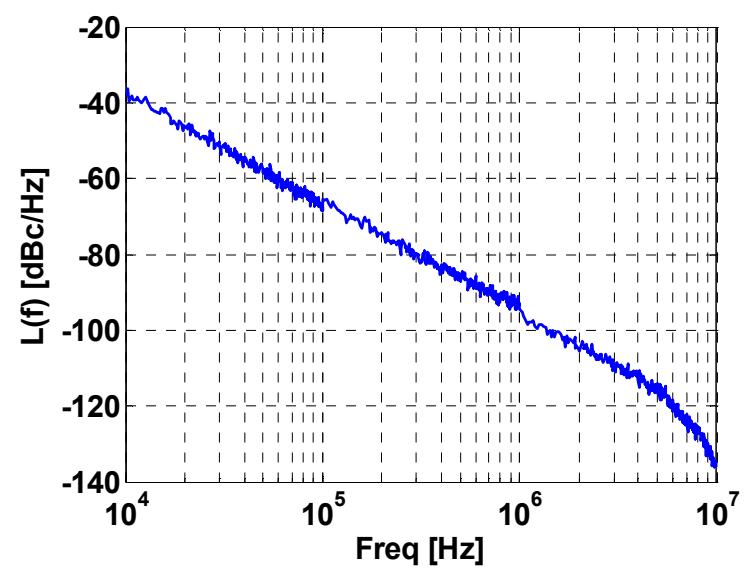

Fig. 6 The phase noise performance @ $1 \mathrm{MHz}$ offset from $64 \mathrm{GHz}$ carrier

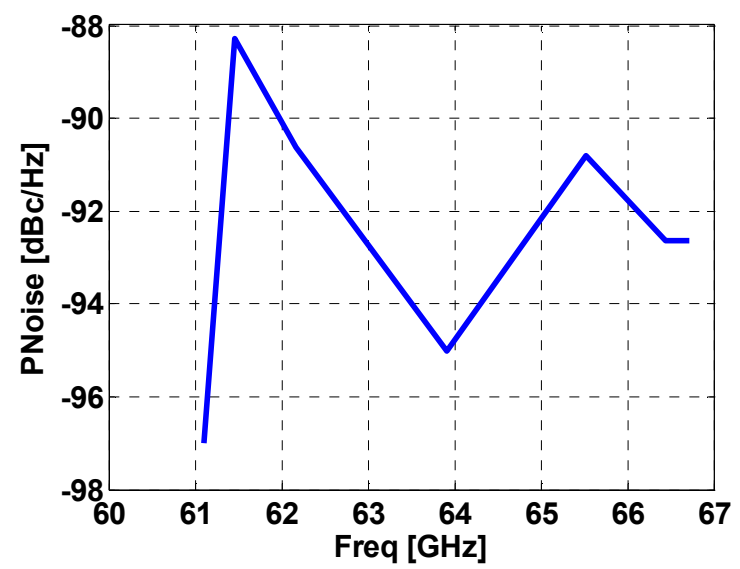

Fig. 7 The phase noise performance $@ 1 \mathrm{MHz}$ offset across the tuning range

\section{CONCLUSION}

A low voltage power efficient mm-wave oscillator using power matching techniques is demonstrated. Using a 90nm CMOS, the oscillator achieves a tuning range from $61.1 \mathrm{GHz}$ to $66.7 \mathrm{GHz}$. Under $0.6 \mathrm{~V}$ supply voltage, the oscillator achieves a phase noise of $-95 \mathrm{dBc} / \mathrm{Hz}$ at $1 \mathrm{MHz}$ offset from $64 \mathrm{GHz}$ carrier while consuming $3.16 \mathrm{~mW}$.
The FOM and $\mathrm{FOM}_{\mathrm{T}}$ are about -186 and -185 respectively. The implemented oscillator achieved the best FOM among mm-wave oscillators.

\section{ACKNOWLEDGEMENT}

The authors wish to acknowledge Ilja Ocket (ESATTELEMIC), Prof. Dominique Schreurs (ESATTELEMIC) and Frederik Daenen (ESAT-MICAS/Imec) for their support during the measurements and Noella Gaethofs (ESAT-MICAS) for the packaging. R\&S Belgium is acknowledged for their excellent support with the measurement setup. Lianming $\mathrm{Li}$ is supported by a Fellowship from the Chinese Scholarship Council.

\section{REFERENCES}

[1] IEEE 802.15 WPAN Millimeter Wave Alternative PHY Task Group 3c, Available: http://www.ieee802.org/15/pub/TG3c.html

[2] C. Doan, S. Emami, A. Nikenejad, and R. Brodersen, "Millimeter-Wave CMOS design," IEEE J. Solid-State Circuits, Vol. 40, no.1, pp. 144-155, Jan.2005.

[3] B. Floyd, "A 16-18.8 GHz sub-integer-N frequency synthesizer for $60 \mathrm{GHz}$ transceiver," IEEE J. Solid-State Circuits, Vol. 43, pp. 1076-1086, May.2008.

[4] L. Li, P. Reynaert, M. Steyaert, "A 90nm CMOS mm-Wave VCO using an LC tank with inductive division," European Solid-State Circuit Conf. pp.238-241, Sept. 2008.

[5] H. Wang, "A $50 \mathrm{GHz}$ VCO in 0.25 um CMOS," ISSCC Digest of Technical Papers, pp. 372-373, Feb. 2001.

[6] M. Tiebout, H.Wohlmuth, and W. Simburger, "A 1V 51GHz fully-integrated VCO in 0.12 um CMOS," ISSCC Digest of Technical Papers, pp. 300-301, Feb. 2002.

[7] F. Ellinger, T. Morf, G. Buren, " $60 \mathrm{GHz}$ VCO with wideband tuning range fabricated in VLSI SOI CMOS technology," IEEE Int. Microwave Symp. Dig., pp. 1329-1332, June. 2004.

[8] C. Cao, K.K.O, "Millimeter-Wave voltage controlled oscillator in 0.13um CMOS technology," IEEE Journal of Solid-State Circuits, Vol. 41, pp. 1297-1304, June.2006.

[9] D. D. Kim, H.Wohlmuth, and W. Simburger, "A $70 \mathrm{GHz}$ manufacturable complementary LC-VCO with $6.14 \mathrm{GHz}$ tuning range in $65 \mathrm{~nm}$ SOI CMOS," ISSCC Digest of Technical Papers, pp. 540-541, Feb. 2007.

[10] J. Borremans, M. Dehan, K. Scheir, M. Kuijk, P. Wambacq, "VCO design for $60 \mathrm{GHz}$ using differential shielded inductors in $0.13 \mu \mathrm{m}$ CMOS" IEEE RFIC Symp., pp. 135138. 2008.

[11] T. Lee, A. Hajimiri, "Oscillator phase noise: a tutorial," IEEE J. Solid-State Circuits, Vol. 35, pp. 326-336, Mar.2000.

[12] Bram De Muer, "Monolithic CMOS fractional-N frequency synthesizers," KU Leuven dissertation pp. 114, 2002. 